\title{
ORIGINAL ARTICLE Expression of DC-SIGN and DC-SIGNRs in placentas of HIV-positive patients
}

\author{
K Pillay, ${ }^{1}$ MB ChB, FCPath (SA), FRCPath(UK), MMed Anat Path; M Cloete, ${ }^{2}$ MB ChB, FCOG (SA), MMed O\&G; \\ H McLeod, ${ }^{1}$ MTech \\ Department of Anatomical Pathology, University of Cape Town, and Red Cross Children's War Memorial Hospital, Cape Town, \\ South Africa \\ ${ }^{2}$ Department of Obstetrics and Gynaecology, Groote Schuur Hospital, University of Cape Town, South Africa
}

Corresponding author: K Pillay (Komala.Pillay@uct.ac.za)

\begin{abstract}
Background. Human dendritic cell-specific intracellular adhesion molecule-3 (ICAM3)-grabbing non-integrin (DC-SIGN) is a mannose-binding lectin that initiates interaction between dendritic cells and resting T-lymphocytes. DC-SIGN is highly expressed in placental tissue on dendritic cells and Hofbauer cells, and it is suggested that HIV may become adsorbed to DC-SIGN on Hofbauer cells as part of the mechanism of mother-to-child HIV transmission. A possible mechanism of transfer of the virus from the Hofbauer cells to the fetus is the subsequent adsorption to DC-SIGN-related molecules (DC-SIGNRs), present on immediately adjacent capillary vascular endothelium. However, data on DC-SIGN and DC-SIGNR expression in the placenta are few.

Methods. Forty term placentas from HIV-positive mothers and 21 term placentas from HIV-negative mothers underwent immunohistochemistry staining for DC-SIGN and DC-SIGNR expression. Five random sets of 10 villi were assessed, and the average number of positive cells were counted in each case. In addition, where possible, maternal and cord blood viral loads and maternal $\mathrm{CD} 4^{+}$counts were performed in the HIV-positive group only.

Results. The median maternal CD4+ count was 377 cells/ $\mu$ l and $27 \%$ of participants had undetectable viral loads; the median detectable viral load was $3.72 \mathrm{log}$. Most (97\%) of the cord bloods tested in infants from HIV-positive mothers had lower than detectable viral loads. HIV-positive cases had significantly greater expression of both DC-SIGNRs (median values in HIV-positive cases, 14.5 positive cells/10 villi (pc/10villi), compared with $11 \mathrm{pc} / 10$ villi in HIV-negative cases, $p=0.020$ ) and DC-SIGN (median value in HIV-positive cases, 26.5 pc/10villi, compared with 23 pc/10villi in HIV-negative cases, $p=0.037$ ). DC-SIGNR expression was also noted in Hofbauer cells and decidual macrophages in addition to endothelium (reported currently). There was no difference in expression of DC-SIGN and DC-SIGNRs in patients with or without chorioamnionitis, but there was an inverse relationship between DC-SIGN and DC-SIGNR expression and maternal CD4 ${ }^{+}$counts in HIV-positive cases.

Conclusion. Both DC-SIGN and DC-SIGNR expression were higher in placentas from HIV-positive mothers compared with HIV-negative cases. These lectins may be potential new therapeutic targets for preventing vertical transmission of HIV.

S Afr J HIV Med 2014;15(3):97-101. DOI:10.7196/SAJHIVMED.1094
\end{abstract}

The rate of vertical transmission of HIV is estimated at between $13 \%$ and $39 \%$ in nonbreastfeeding women. ${ }^{[1]}$ Most of these cases occur during the intrapartum period during vaginal delivery. However, a small percentage results from HIV infection across the placenta (1 - 2\%). A possible mechanism for late-gestation HIV transmission may be maternal-fetal transfusions during labour, which is significantly lower in elective caesarean sections (CSs). ${ }^{[2]}$

Reverse transcriptase inhibitors (azidothymidine/zidovudine (AZT) and lamivudine) rapidly cross the placenta, and cord blood levels equal or exceed those in the maternal circulation at the time of delivery. ${ }^{[3]}$ Even a single dose of nevirapine, when administered during labour and to an infant after birth, can reduce vertical transmission by $50 \%$ because it rapidly crosses the placenta. ${ }^{[3,4]}$ The use of highly active antiretroviral therapy (HAART) throughout pregnancy and prophylactic CS have reduced the rate of vertical transmission in the US to $<2 \%$. ${ }^{[5]}$
Infants with a positive HIV polymerase chain reaction (PCR) test within 48 hours of birth are considered to have been infected in utero. However, infants who tested HIV-negative at birth but tested positive at 6 weeks or 12 weeks postnatally with PCR testing are considered to have been infected intrapartum or immediately post partum. ${ }^{[6]}$ In addition, HIV has been demonstrated in second-trimester fetuses and second-trimester cord bloods with associated placental HIV infection supporting in utero transmission. ${ }^{[7-9]}$

Factors that may increase the risk of mother-to-infant transmission include advanced HIV-related illness, low CD4 counts, high HIV-1 viral loads (VLs), the presence of sexually transmitted diseases, viral phenotype, premature rupture of membranes, chorioamnionitis and absence of maternal autologous neutralising antibodies. ${ }^{[2]}$ But even at the highest VLs, only about half of exposed infants become infected with HIV. ${ }^{[2,4]}$

It has been proposed that a breach in the trophoblast layer lining the chorionic villi may result in contact of Hofbauer cells 
(specialised fetal macrophages) with maternal blood and amniotic fluid. There may also be entry of HIV into the trophoblast layer with subsequent infection of the cell, or the virus could traverse the cells intact, following release at the basolateral surface and exposure to stromal cells. The two pathways are not mutually exclusive. ${ }^{[2]}$ However, trophoblast cells are reported to be only 'moderately susceptible' to HIV infection in vitro. ${ }^{\left[{ }^{[10]}\right.}$ It has been shown that tumour necrosis factor alpha $(\mathrm{TNF} \alpha)$ significantly upregulated transcytosis of HIV-1 across the trophoblast layer whereas an anti-inflammatory drug (tenidap) significantly reduced transcytosis rates. ${ }^{[5]}$

Infection of trophoblast cells remains controversial. ${ }^{[11]}$ C-C chemokine receptor type 5 (CCR5) and C-X-C chemokine receptor type 4 (CXCR4) were demonstrated on placental macrophages and lymphocytes, but not in trophoblast cells.

Mattern et al. ${ }^{[12]}$ demonstrated p24 antigen within scattered Hofbauer cells but not within trophoblast cells or vascular endothelium, in 5/19 term placentas (26\%) from HIV-positive women. There was no association between p24 antigen detection and vertical transmission. ${ }^{[12]}$ Another study showed positive Hofbauer cell staining for p24 in 4/9 (44\%) placentas from HIV-positive mothers, but there was also decidual macrophage $(n=1)$, intermediate trophoblast $(n=1)$ and villous endothelium $(n=1)$ positivity. ${ }^{[13]}$

Human dendritic cell-specific intracellular adhesion molecule-3 (ICAM3)-grabbing non-integrin (DC-SIGN) is a type II mannose-binding lectin that initiates interaction between dendritic cells and resting T-lymphocytes. It is highly expressed in dendritic cells on mucosal tissue and has also been found to be highly expressed in placental tissue on maternal decidual cells and fetal Hofbauer cells. ${ }^{[14]}$ It has been proposed that HIV may become adsorbed to DC-SIGN on Hofbauer cells and/or infect Hofbauer cells. Various mechanisms have been proposed that may allow transfer of the virus from the Hofbauer cells to the fetus.

One mechanism includes subsequent adsorption to DC-SIGN-related molecules (DC-SIGNRs) present on immediately adjacent capillary vascular endothelium. ${ }^{[14]}$ A DC-SIGNR is a transmembrane lectin that binds mannose residues including the glycans of ICAM3 and HIV-1 and HIV-2. It is prominently expressed on endothelial cells derived from liver sinusoids, lymph node sinuses and placenta. ${ }^{[15]}$ However, the possible role of DC-SIGN and DC-SIGNRs in vertical transmission of HIV is still poorly understood.

The purpose of this study was to assess the expression of DC-SIGN and DC-SIGNRs in placentas of HIV-positive patients with the following objectives: to compare the expression of DC-SIGN and DC-SIGNRs in placentas of HIV-positive and HIV-negative patients; to correlate the expression of DC-SIGN and DC-SIGNRs with VLs, history of antiretroviral therapy and evidence of in-utero HIV transmission (if possible); and to assess the placentas from HIV-positive patients for pathology, including chorioamnionitis and the presence of specific infective agents.

\section{Methods}

Forty placentas from HIV-positive patients at term gestation were collected from the Department of Obstetrics and Gynaecology, Mowbray Maternity Hospital, Cape Town, South Africa. In addition, 21 placentas from HIV-negative patients were collected. Ethical approval for the study was granted by the Faculty of Health Sciences at the University of Cape Town and informed consent for research was obtained from the patients (REC REF 290/2005).

The research included histology and immunohistochemistry of the placentas, performed in the Division of Anatomical Pathology, Groote Schuur Hospital, Cape Town. The placentas were fixed in $10 \%$ buffered formalin and analysed in the postmortem laboratory. The analysis included macroscopic description (with plate weight), serial sectioning and taking of three blocks, depending on the presence of macroscopic abnormality, which included umbilical cord, membranes, cord insertion site and a peripheral section of placenta.

Haematoxylin and eosin stained sections were analysed by K Pillay and a placental histopathology report was issued. One suitable block was selected for immunohistochemistry. Two micrometre sections were cut onto poly-L-lysine coated slides and stained with DC-SIGN (purified mouse antihuman CD209 antibody, dilution 1:40, BD Biosciences, US) and DC-SIGNRs (monoclonal antihuman CD209L antibody, dilution 1:40, R\&D systems, US) using the Envision kit. Diaminobenzidine (brown) was used as chromogen. Positive and negative controls were run simultaneously.

Five random sets of 10 villi were assessed and an average number of positive cells were counted in each case. Maternal and fetal VLs, with blood taken at the time of delivery, were performed for the HIV-positive group in the Department of Virology, Groote Schuur Hospital, Cape Town.

Data were analysed using Stata Version 10.0 (Stata Corporation, US). Median values of $\mathrm{CD}^{+}$cell count, HIV VL and DC-SIGNR expression were compared using the Wilcoxon rank-sum test; proportions were compared using Fisher's exact test; and correlations were analysed using Spearman's rho.

\section{Results}

Forty term placentas from HIV-positive mothers (mean age (range) 26 (14 - 38) years) and 21 term placentas from HIV-negative mothers (28 (16 - 40) years) were assessed.

All HIV-positive mothers received antiretrovirals for the prevention of motherto-child transmission (PMTCT). The majority started AZT monotherapy in the third trimester $(85 \%, n=34), 2$ were on AZT and nevirapine or lamivudine (5\%) and 4 were on HAART before pregnancy (10\%). Two of the $40 \mathrm{HIV}$-positive patients tested positive for syphilis (Venereal Disease Research Laboratory (VDRL)-positive) and received treatment for this, while none of the HIVnegative mothers was VDRL-positive.

Most of the deliveries were by CS in both groups (Table 1). There were 2/38 (5\%) small for gestational age (SGA) babies from HIV-positive mothers, one with a history of gestational proteinuric hypertension even though the placenta was histologically normal. There were 3/21 (14\%) SGA babies from the HIV-negative cases, two of whom showed ischaemic changes histologically.

Table 1. Antenatal history and delivery

\begin{tabular}{llll}
\hline HIV status & VDRL-positive & Delivery & SGA \\
\hline HIV-positive $(n=40)$ & 2 & $10 \mathrm{NVD}$ & 2 \\
& $30 \mathrm{CS}$ & \\
HIV-negative $(n=21)$ & 0 & $5 \mathrm{NVD}$ & 3 \\
& $16 \mathrm{CS}$ & \\
$\begin{array}{l}\text { VDRL = Venereal Disease Research Laboratory; NVD = normal vaginal delivery; CS = caesarean section; } \\
\text { SGA = small for gestational age. }\end{array}$ &
\end{tabular}


In the HIV-positive group, the median maternal $\mathrm{CD}^{+}$count was 324.5 cells $/ \mu \mathrm{l}$ (interquartile range 246 - 522) $(n=38)$ and 27\% of HIV-positive participants had undetectable VLs; the median detectable VL was $3.59 \mathrm{log}$ $(n=33)$.

In infants from HIV-positive mothers, $97 \%$ of the cord bloods tested had lower than detectable VLs (30/31). The mother of the infant with the positive cord blood $(n=1)$ had higher than median VL, lower than median $\mathrm{CD}^{+}$count, received AZT, and the placenta

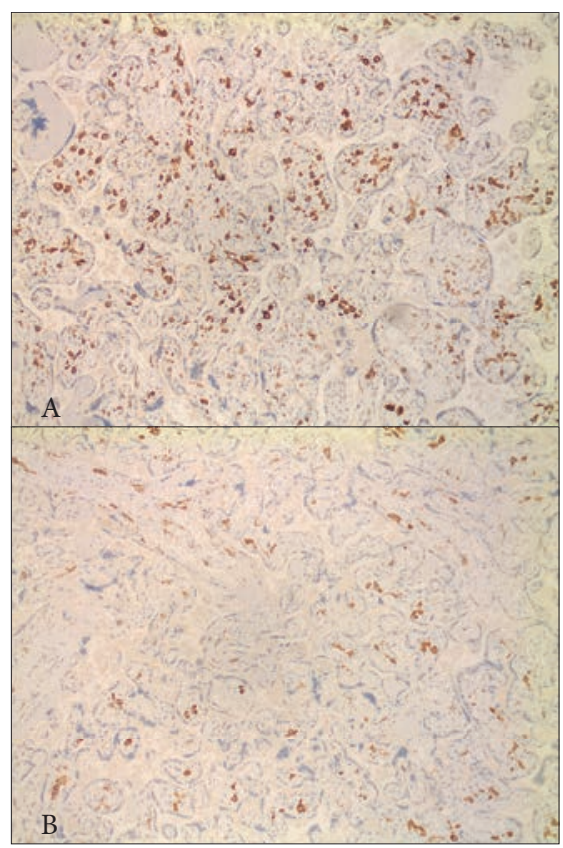

Fig. 1. A. DC-SIGN expression in Hofbauer cells in an HIV-positive case with chorioamnionitis and a $C D 4^{+}$count of 118 cells/ul; B. DC-SIGN expression in Hofbauer cells in an HIV-negative case. (DCSIGN = human dendritic cell-specific intracellular adhesion molecule-3 (ICAM3)-grabbing nonintegrin.)

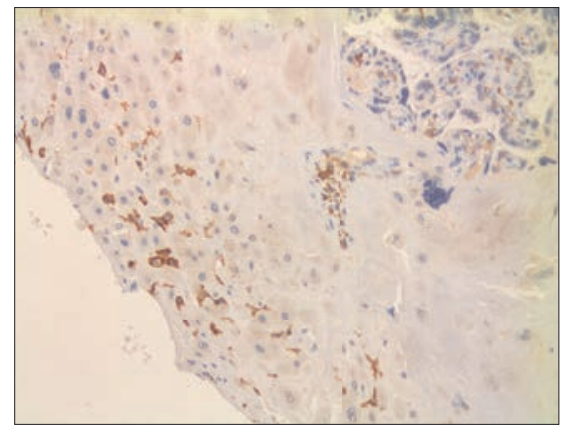

Fig. 2. DC-SIGN expression in decidual macrophages in an HIV-positive case with the only detectable viral load in the cord blood. (DCSIGN = human dendritic cell-specific intracellular adhesion molecule-3 (ICAM3)-grabbing nonintegrin.) showed chorioamnionitis with cord-vessel vasculitis and chronic deciduitis.

In the HIV-positive group, 15 placentas showed chorioamnionitis (grade $1 n=7$, grade $2 n=6$, grade $3 n=2$; stage $1 n=3$, stage 2 $n=9$, stage $3 n=3)$. There was also plate-vessel vasculitis $(n=9)$, cord-vessel vasculitis $(n=7)$ and funisitis $(n=2)$. In the HIV-negative group, there were three cases of chorioamnionitis (grade $2 n=2$, grade $3 n=1$; stage $1 n=1$, stage $3 n=2$ ) and two cases of mild cord-vessel vasculitis with no evidence of plate-vessel vasculitis or funisitis in this group.

There was no significant difference in the incidence of chorioamnionitis between placentas from HIV-positive and HIVnegative mothers $(p=0.084)$. There were 9 cases of chronic deciduitis, 7 in HIVpositive placentas and 2 in HIV-negative patients $(p=0.704)$. There was no evidence of villitis or intervillositis, and no opportunistic infections (e.g. cytomegalovirus inclusions, toxoplasmosis or cryptococcosis) were noted on morphology.

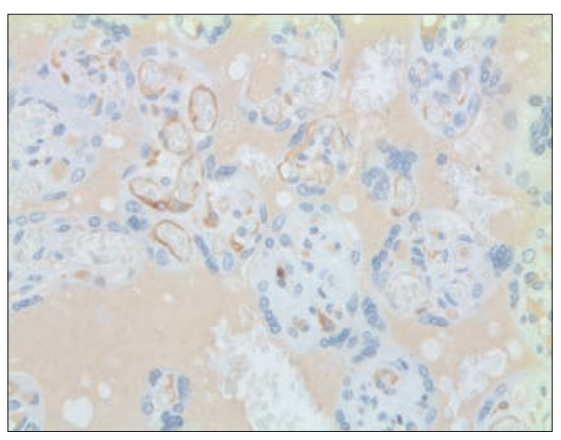

Fig. 3. DC-SIGNR expression in endothelial cells and Hofbauer cells in an HIV-positive case with maternal $C D 4^{+}$count of 365 cells/ $\mu$ l and a viral load of 3.84 log. (DC-SIGNR = human dendritic cellspecific intracellular adhesion molecule-3 (ICAM3)grabbing non-integrin-related molecule.)

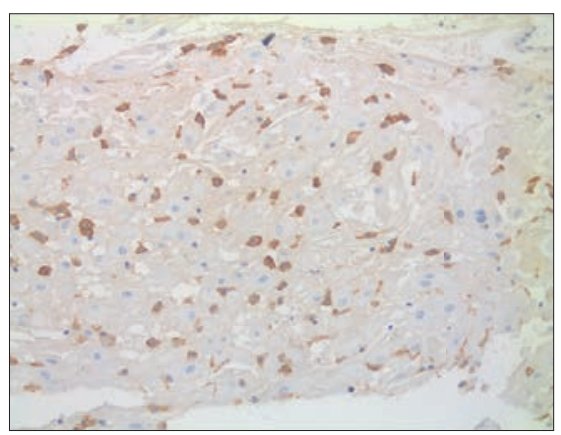

Fig. 4. DC-SIGNR expression in decidual macrophages in an HIV-positive case with maternal CD4 $4^{+}$count of 45 cells/ $\mu$ l. (DC-SIGNR = human dendritic cell-specific intracellular adhesion molecule-3 (ICAM3)-grabbing non-integrinrelated molecule.)
Additional features that were noted on histology in the HIV-positive group included evidence of meconium exposure $(n=10)$, focal infarct $(n=1)$, focal decidual vasculopathy $(n=1)$, dysmaturity $(n=3)$, fetal thrombotic vasculopathy $(n=1)$, chorangiosis $(n=1)$, intervillous thrombus $(n=2)$ and intervillous haemorrhage $(n=3)$; and in the HIV-negative group, infarcts $(n=2)$, decidual vasculopathy $(n=1)$ and dysmaturity $(n=2)$.

DC-SIGN expression was noted in Hofbauer cells (Fig. 1) and decidual macrophages (Fig. 2) while DC-SIGNR expression was seen in endothelial cells (Fig. 3), some Hofbauer cells and decidual macrophages (Fig. 4). Decidual macrophage staining for DC-SIGNRs has not been described previously in the literature.

HIV-positive cases had significantly greater expression of both DC-SIGNRs (median values in $\mathrm{HIV}$-positive cases, 14.5 positive cells/10 villi (pc/10villi), compared with $11 \mathrm{pc} / 10$ villi in HIV-negative cases, $p=0.020$ ) and DC-SIGN (median values in HIVpositive cases, $26.5 \mathrm{pc} / 10 \mathrm{villi}$, compared with $23 \mathrm{pc} / 10$ villi in HIV-negative cases, $p=0.037$ ).

In addition, the expression of DC-SIGN and DC-SIGNRs was inversely associated with $\mathrm{CD}^{+}$count in HIV-positive cases $(p<0.05$ for both, Fig. 5) but the relationship with maternal VL was not statistically significant. There was no significant difference in the expression of DC-SIGN $(p=0.833)$ and DC-SIGNRs $(p=0.557)$ in placentas with or without placental membrane inflammation. There was also no association with mode of delivery (normal vaginal delivery v. CS).

\section{Discussion}

In the placenta, no co-expression of DC-SIGN and DC-SIGNRs was detected previously. ${ }^{[15]}$ DC-SIGNR expression was detected only on capillary endothelial cells, whereas DCSIGN was expressed only by Hofbauer cells. ${ }^{[15]}$ However, Mummidi et al. ${ }^{[16]}$ demonstrated expression of DC-SIGN on placental capillary endothelium as well. DC-SIGNRs showed a low level of expression in the endometrium. ${ }^{[17]}$ In this study, we demonstrated DC-SIGNR expression on Hofbauer cells and decidual cells as well.

DC-SIGN and DC-SIGNR lectins have been shown to mediate infection in cis (cells with DC-SIGN receptors e.g. dendritic cells) and trans (e.g. T-lymphocytes) cells. ${ }^{[18]}$ Currently, it is felt that DC-SIGN and DC-SIGNRs represent the best candidate molecules for mediating intrauterine transmission. ${ }^{[10]}$ DC-SIGN and DC-SIGNRs appear to function 

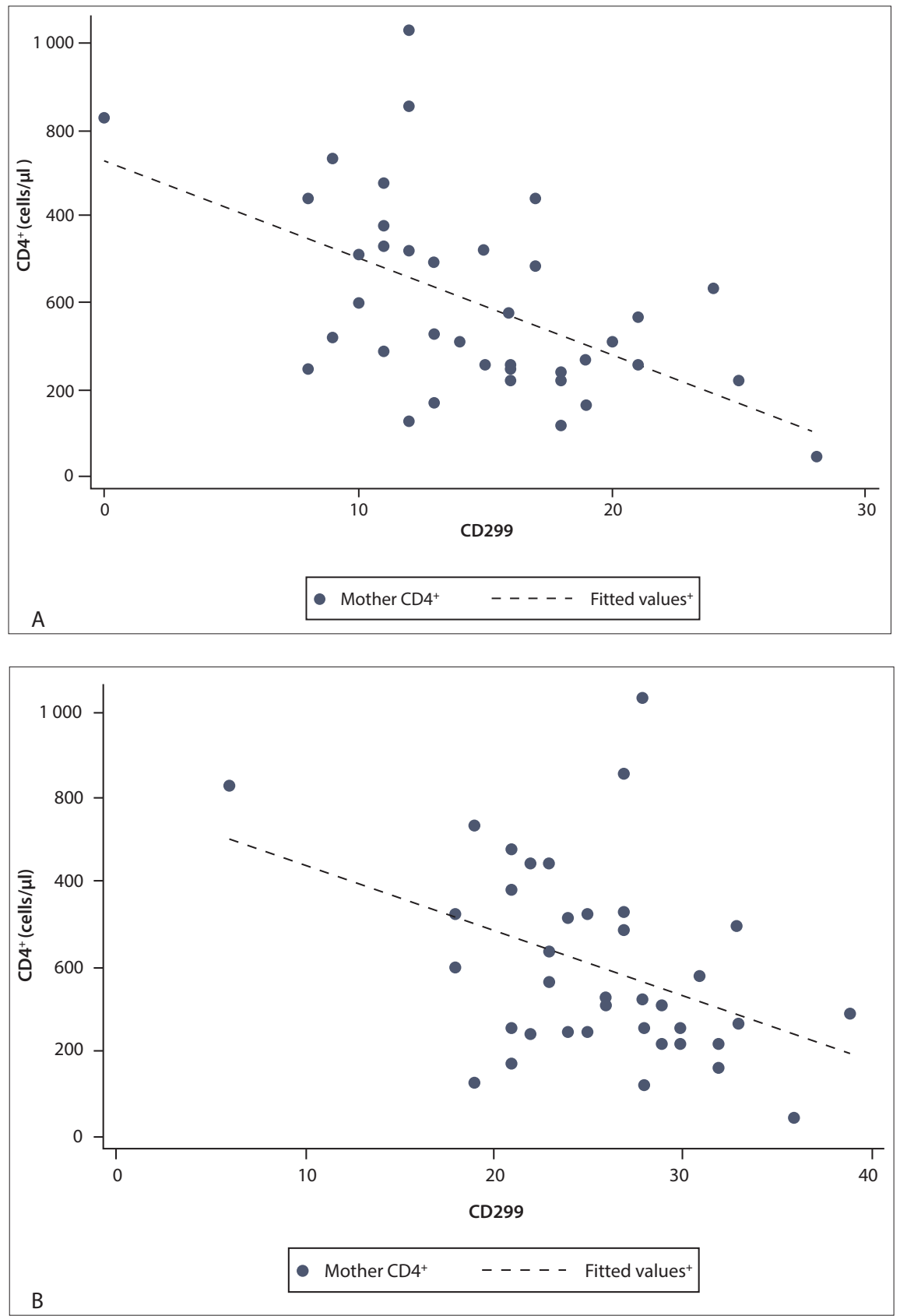

Fig. 5. A. CD4+ v. DC-SIGNRs (CD299) showing a statistically significant inverse correlation, the higher the expression, the lower the CD4+ count; B. CD4 ${ }^{+}$v. DC-SIGN (CD209) showing a statistically significant inverse correlation, the higher the expression, the lower the CD4+ count. (DC-SIGNR = human dendritic cell-specific intracellular adhesion molecule-3 (ICAM3)-grabbing non-integrin-related molecules.)

as universal attachment factors for primate lentiviruses, namely HIV-1, HIV-2 and simian immunodeficiency viruses. ${ }^{[19]}$ However, research in this field is still in progress and many studies are in vitro in nature. One such study has shown a decrease in DC-SIGN expression following infection with $\mathrm{HHV}-6 .{ }^{[20,21]}$ According to these papers, this may apply to the infection of HIV-1, or DC-SIGN expression may be up-regulated in the setting of HIV-1. Another in-vitro study has shown that while DC-SIGN is up-regulated by type 1 and type 2 blocking molecules. Other lectin receptors include mannose receptor and langerin expressed on intradermal dendritic cells and Langerhans cells (DC-SIGN negative), respectively, which may have HIV-binding affinity. ${ }^{[23]}$ DC-SIGN on dendritic cells has been shown to bind and transfer HIV through its interaction with HIV gp120. Disruption of this interaction represents a potential new therapeutic approach for preventing HIV transmission. ${ }^{[24]}$

Gurney et al. ${ }^{[24]}$ found that DC-SIGNpositive dendritic cells in the submucosa may be responsible for the transfer of HIV-1 from the periphery to the draining lymph nodes during primary sexual mucosal transmission of HIV-1. DC-SIGN-positive cells appear to be immature dendritic cells in that they did not express the dendritic cell maturation molecule CD83. The Hofbauer cells have also been shown to be human leukocyte antigen (HLA)

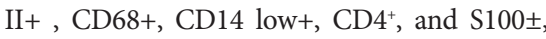
CD86- and CMRF-44-. ${ }^{[23,25]}$

DC-SIGN is also expressed by some decidual cells with CD14high+ and S100-negative phenotype compared with the Hofbauer cells. DC-SIGN-positive Hofbauer cells co-express $\mathrm{CD}^{+}$and chemokine receptors CCR-5 and CXCR4. ${ }^{[23]}$ Gurney et al. ${ }^{[24]}$ have demonstrated an increase in DC-SIGN-positive cells in the gut mucosa of HIV-positive patients (two - fourfold greater). Increased DC-SIGN expression in the gut mucosa correlated with a type 2 environment (increased interleukin (IL)-10/ IL-12 ratio) and a decrease in the levels of the costimulatory molecules CD86 and CD80.

Micro-array analysis experiments have shown that IL-10-induced dendritic cells result in a DC-SIGN high-expressing subset. There is a shift in pregnancy towards the production of T-helper 2 (Th2) cytokines, including IL-4 and IL-10, promoted by progesterone. $^{[4]}$ The Th2 cytokines IL-4 and IL-13 play an important role in inducing DC-SIGN expression under specific conditions. ${ }^{[23]}$ However, it has been shown that placentas from non-transmitting women maintain normal type 2 placental cytokines (IL-4, IL-10) whereas transmitting women have placentas that express type 1 cytokines (interferon-gamma, tumour necrosis factor beta). ${ }^{[1]}$ Other cytokines (TNFa, IL-6, etc.) stimulate the HIV infection and facilitate transmission during pregnancy. ${ }^{[2]}$

In this study, we found an increase in DC-SIGN expression in placentas from HIVpositive patients, which may be due to the increased cytokine milieu. 
It has been shown that an anti-DC-SIGN antibody blocked virus binding to dendritic cells by almost $90 \%$, with low viral inocula close to those found in seminal fluid from untreated HIV-positive patients. ${ }^{[24]}$ In addition, when the HIV was exposed to the total mucosal mononuclear cells, about 40-fold more viruses were bound to the DC-SIGN-positive population compared with the total gut mononuclear cell population. Therefore, more than $90 \%$ of the bound virus was associated with the DC-SIGN-positive cells, which only constitute about $1-5 \%$ of the total mucosal mononuclear cell population. DC-SIGN is therefore a potential target for therapeutic intervention to reduce viral transmission (after sexual transmission and during vertical transmission). ${ }^{[1,24]}$ The ability of DC-SIGN to enhance infection markedly when virus levels are low may be of particular importance in the setting of prophylaxis against mother-to-child transmission and HAART. ${ }^{[26]}$

Placental membrane inflammation comprises histological evidence of chorioamnionitis and/or funisitis with or without associated vasculitis. Plasma cell (chronic) deciduitis is demonstrated by the presence of plasma cells in the maternal decidua. ${ }^{[27]}$

Jauniaux et al ${ }^{[28]}$ demonstrated a high incidence of chorioamnionitis (43\%) in placentas from HIV-positive women, but villitis was absent. According to the study by Schwartz et al. ${ }^{[29]}$ no histopathological finding occurred with increased frequency in placentas of transmitting women even though there was a trend towards increased frequency of plasma cell deciduitis. Only 1/17 transmitting women had a placenta with low-grade neutrophilic inflammation. More uninfected women had villitis. ${ }^{[29]}$ Chandwani et al. ${ }^{[30]}$ demonstrated no cases of villitis and a higher incidence of chorionitis in placentas from HIV-positive women and p24 antigen within trophoblastic cells, contrary to other studies. ${ }^{[30]}$

In our study, we demonstrated no significant difference in placental membrane inflammation or chronic deciduitis between HIV-positive and HIV-negative women. There were no cases of villitis or intervillositis in any of the cases. In addition, there was no association of DC-SIGN or DC-SIGNR expression with placental membrane inflammation.

\section{Conclusion}

Both DC-SIGN and DC-SIGNR expression were higher in placentas from HIV-positive mothers compared with HIV-negative cases, and this was statistically significant. DC-SIGNR expression was also noted in Hofbauer cells and decidual macrophages in addition to endothelium (reported currently). There was no association of DC-SIGN or DC-SIGNR expression with the presence of placental membrane inflammation, but there was a statistically significant inverse relationship between DC-SIGN and DC-SIGNR expression and maternal CD4+ counts in HIV-positive cases. There was possible in utero transmission of HIV in one of the $40 \mathrm{HIV}$-positive cases (3\%).

\section{References}

1. Kourtis AP, Lee FK, Abrams EJ, et al. Mother-to-child transmission of HIV-1: Timing and implications for prevention. Lancet Infect Dis 2006;6(11):726-732. [http://dx.doi.org/10.1016/s1473-3099(06)70629-6]

2. Anderson V, Carneiro M, Bulterys M, et al. Perinatal infections: HIV and coinfections in the placenta and therapeutic interventions - a workshop report Placenta 2001; 22(Suppl A):S34-37. [http://dx.doi.org/10.1053/plac.2001.0641]

3. Mirochnick M. Antiretroviral pharmacology in pregnant women and their newborns Ann N Y Acad Sci 2000;918:287-297. [http://dx.doi.org/10.1111/j.1749-6632.2000 tb05498.x]

4. Spector SA. Mother-to-infant transmission of HIV-1: The placenta fights back. J Clin Invest 2001;107(3):267-269. [http://.dx.doi.org/10.1172/JCI12094]

5. Parry S, Zhang J, Koi H, et al. Transcytosis of human immunodeficiency virus 1 across the placenta is enhanced by treatment with tumour necrosis factor alpha. J Gen Virol 2006;87(Pt8):2269-2278. [http://dx.doi.org/10.1099/vir.0.81071-0]
6. Bryson YJ, Luzuriaga K, Sullivan JL, Wara DW. Proposed definitions for in utero versus intrapartum transmission of HIV-1. N Engl J Med 1992;327(17):1246-1247. 7. Mano H, Chermann JC. Fetal human immunodeficiency virus type 1 infection of different organs in the second trimester. AIDS Res Hum Retroviruses 1991;7(1):83-88.

8. Maury W, Potts BJ, Rabson AB. HIV-1 infection of first-trimester and term human placental tissue: a possible mode of maternal-fetal transmission. J Infect Dis 1989;160(4):583-588. [http://dx.doi.org/10.1093/infdis/160.4.583]

9. De Andreis C, Simoni G, Rossella F, et al. HIV-1 proviral DNA polymerase chain reaction detection in chorionic villi after exclusion of maternal contamination by variable number of tandem repeats analysis. AIDS 1996;10(7):711-715. [http:// dx.doi.org/10.1097/00002030-199606001-00004]

10. Soilleux EJ, Coleman N. Transplacental transmission of HIV: a potential role for HIV binding lectins. Int J Biochem Cell Biol 2003;35(3):283-287. [http://dx.doi. org/10.1016/S1357-2725(02)00132-2]

11. Behbahani H, Popek E, Garcia P, et al. Up-regulation of CCR5 expression in the placenta is associated with human immunodeficiency virus-1 vertical transmission. Am J Pathol 2000;157(6):1811-1818. [http://dx.doi.org/10.1016/ S0002-9440(10)64819-5]

12. Mattern CF, Murray K, Jensen A, et al. Localization of human immunodeficiency virus core antigen in term human placentas. Pediatrics 1992;89(2):207-209.

13. Martin AW, Brady K, Smith SI, et al. Immunohistochemical localization of human immunodeficiency virus p24 antigen in placental tissue. Hum Pathol 1992;23(4):411-414. [http://dx.doi.org/10.1016/0046-8177(92)90088-K]

14. Geijtenbeek TB, van Vliet SJ, van Duijnhoven GC, et al. DC-SIGN, a dentritic cellspecific HIV-1 receptor present in placenta that infects T cells in trans: A review. Placenta 2001;22(Suppl A):S19-23. [http://dx.doi.org/10.1053/plac.2001.0674]

15. Soilleux EJ, Morris LS, Rushbrook S, et al. Expression of human immunodeficiency virus (HIV)-binding lectin DC-SIGNR: Consequences for HIV infection and immunity. Hum Pathol 2002;33(6):652-659. [http://dx.doi.org/10.1053/ hupa.2002.124036]

16. Mummidi S, Catano G, Lam L, et al. Extensive repertoire of membrane-bound and soluble dendritic cell-specific ICAM-3-grabbing nonintegrin 1 (DC-SIGN1) and DC-SIGN2 isoforms. Inter-individual variation in expression of DC-SIGN transcripts. J Biol Chem 2001;276(36):33196-33212. [http://dx.doi.org/10.1074/ jbc.M009807200]

17. Soilleux EJ, Barten R, Trowsdale J. DC-SIGN; a related gene, DC-SIGNR; and CD23 form a cluster on 19p13. J Immunol 2000;165(6):2937-2942. [http://dx.doi. org/10.4049/jimmunol.165.6.2937]

18. Gummuluru S, KewalRamani VN, Emerman M. Dendritic cell-mediated viral transfer to $\mathrm{T}$ cells is required for human immunodeficiency virus type 1 persistence in the face of rapid cell turnover. J Virol 2002;76(21):10692-10701. [http://dx.doi. org/10.1128/JVI.76.21.10692-10701.2002]

19. Pohlmann S, Soilleux EJ, Baribaud F, et al. DC-SIGNR, a DC-SIGN homologue expressed in endothelial cells, binds to human and simian immunodeficiency viruses and activates infection in trans. Proc Natl Acad Sci USA 2001;98(4):26702675. [http://dx.doi.org/10.1073/pnas.051631398]

20. Niiya H, Azuma T, Jin L, et al. Transcriptional downregulation of DC-SIGN in human herpesvirus 6-infected dendritic cells. J Gen Virol 2004;85(Pt 9):2639-2642. [http://dx.doi.org/10.1099/vir.0.80095-0]

21. Chehimi J, Luo Q, Azzoni L, et al. HIV-1 transmission and cytokine-induced expression of DC-SIGN in human monocyte-derived macrophages. J Leukoc Biol 2003;74(5):757-763. [http://dx.doi.org/10.1189/jlb.0503231]

22. Wu L, Martin TD, Vazeux R, et al. Functional evaluation of DC-SIGN monoclonal antibodies reveals DC-SIGN interactions with ICAM-3 do not promote human immunodeficiency virus type 1 transmission. J Virol 2002;76(12):5905-5914. [http://dx.doi.org/10.1128/JVI.76.12.5905-5914.2002]

23. Soilleux EJ. DC-SIGN (dendritic cell-specific ICAM-grabbing non-integrin) and DC-SIGN-related (DC-SIGNR): Friend or foe? Clin Sci (Lond) 2003;104(4):437446. [http://dx/doi.org/10.1042/CS20020092]

24. Gurney KB, Elliott J, Nassanian H, et al. Binding and transfer of human immunodeficiency virus by DC-SIGN+ cells in human rectal mucosa. J Virol 2005;79(9):5762-5773. [http://dx.doi.org/10.1128/JVI.79.9.5762-5773.2005]

25. Bockle BC, Solder E, Kind S, et al. DC-SIGN+ CD163+ macrophages expressing hyaluronan receptor LYVE-1 are located within chorion villi of the placenta. Placenta 2008;29(2):187-192. [http:dx.doi.org/10.1016/j.placenta.2007.11.003]

26. Soilleux EJ, Morris LS, Lee B, et al. Placental expression of DC-SIGN may mediate intrauterine vertical transmission of HIV. J Pathol 2001;195(5):586-592.[http:// dx.doi.org/10.1002/path.1026]

27. Wabwire-Mangen F, Gray RH, Miro FA, et al. Placental membrane inflammation and risks of maternal-to-child transmission of HIV-1 in Uganda. J Acquir Immune Defic Syndr 1999;22:379-385. [http://dx.doi.org/10.1097/00126334-199912010-00009]

28. Jauniaux E, Nessmann C, Imbert MC, et al. Morphological aspects of the placenta in HIV pregnancies. Placenta 1988;9(6):633-642. [http://dx.doi.org/10.1016/01434004(88)90007-0]

29. Schwartz DA, Sungkarat S, Shaffer N, et al. Placental abnormalities associated with human immunodeficiency virus type 1 infection and perinatal transmission in Bangkok, Thailand. J Infect Dis 2000;182(6):1652-1657. [http://dx.doi. org/10.1086/317634]

30. Chandwani S, Greco MA, Mittal K, et al. Pathology and human immunodeficiency virus expression in placentas of seropositive women. J Infect Dis 1991;163(5):11341138. [http://dx.doi.org/10.1093/infdis/163.5.1134] 